\title{
White-Box Watermarking Scheme for Fully-Connected Layers in Fine-Tuning Model
}

\author{
Minoru Kuribayashi, Takuro Tanaka, Shunta Suzuki, Tatsuya Yasui, Nobuo Funabiki \\ Okayama University \\ Okayama, Japan \\ kminoru@okayama-u.ac.jp
}

\begin{abstract}
For the protection of trained deep neural network(DNN) models, embedding watermarks into the weights of the DNN model have been considered. However, the amount of change in the weights is large in the conventional methods, and it is reported that the existence of hidden watermark can be detected from the analysis of weight variance. This helps attackers to modify the watermark by effectively adding noise to the weight. In this paper, we focus on the fully-connected layers of fine-tuning models and apply a quantization-based watermarking method to the weights sampled from the layers. The advantage of the proposed method is that the change caused by watermark embedding is much smaller and the distortion converges gradually without using any loss function. The validity of the proposed method was evaluated by varying the conditions during the training of DNN model. The results shows the impact of training for DNN model, effectiveness of the embedding method, and high robustness against pruning attacks.
\end{abstract}

\section{CCS CONCEPTS}

- Security and privacy $\rightarrow$ Digital rights management; $\bullet$ Computing methodologies $\rightarrow$ Neural networks; • Information systems $\rightarrow$ Multimedia information systems.

\section{KEYWORDS}

Watermark; fine-tuning; QIM; local minima; convergence

\section{ACM Reference Format:}

Minoru Kuribayashi, Takuro Tanaka, Shunta Suzuki, Tatsuya Yasui, Nobuo Funabiki. 2021. White-Box Watermarking Scheme for Fully-Connected Layers in Fine-Tuning Model. In Proceedings of the 2021 ACM Workshop on Information Hiding and Multimedia Security (IH\&MMSec '21), fune 22-25, 2021, Virtual Event, Belgium. ACM, New York, NY, USA, 6 pages. https: //doi.org/10.1145/3437880.3460402

\section{INTRODUCTION}

Since DNN models have high intellectual property rights, it is necessary to protect them from unauthorized users by restricting access to the model's architecture and hyperparameters. As a countermeasure against unauthorized copying of trained DNN models, secret information is inserted into the parameters of a DNN model

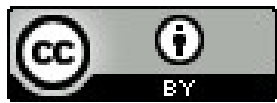

This work is licensed under a Creative Commons Attribution International 4.0 License. IH\&MMSec '21, June 22-25, 2021, Virtual Event, Belgium.

(C) 2021 Copyright held by the owner/author(s).

ACM ISBN 978-1-4503-8295-3/21/06.

https://doi.org/10.1145/3437880.3460402 being trained so that one can prove his/her authorship on the model $[1,4,12,14,16]$. Compared to watermarking techniques for multimedia content, watermarking for DNN models poses a new technical challenge because it is a functionality of the model trained for a specific task that needs to be protected.

\subsection{Related Works}

In $[12,16]$, a binary sequence of watermark information is embedded into the weight parameters of a convolutional neural network (CNN) model selected from its specified layer. The weight parameters are updated at each learning process so that the multiplication with a secret matrix approaches to the watermark. The degradation of the model caused by the embedding operation depends on the properties of the secret matrix. The operation is performed in parallel with the training of the model by using a supervised dataset. As the loss function, binary cross entropy is used to reduce the degradation of the performance of $\mathrm{CNN}$-based system. In addition to the cost function of the original model, the embedding loss function is considered in the training so that the weights are updated according to the watermark as well as the supervised dataset. In $[4,14]$, the selection of feature vector is refined from the method in $[12,16]$. However, a vulnerability is reported in [17]. After embedding the watermark, the variance of the weight values becomes large, and the information of the message hidden in one CNN layer is leaked. It has also been shown that the watermark is easily modified by overwriting. The other interesting approach is based on a visible watermarking method presented in [18]. Similar to the case of adversarial examples, the watermarked image is classified into a different label due to the embedded visible watermark. The drawback is the less robustness against the same watermark removal attacks that have been investigated for the last few decades.

Generally, the weight parameters of neural networks are initialized before learning process and refined to reach a single local minimum after a series of epochs. Several researchers have noticed that, while multi-layered neural networks have many local minima, the result of multiple experiments consistently give very similar performance [6, 7]. For deeper or larger models, the loss values are almost the same, so the local minimum is sufficient. Furthermore, as the model gets more complex, there are losses which are closer to the global minimum. This implies that by choosing such local minima, we can embed watermarks in DNN models without degrading the performance.

\subsection{Our Contribution}

In this paper, we propose a novel watermarking method for DNN models by modifying initial values for the weights specified by watermark information. Before the learning process, the weight 
parameters are randomly selected based on a secret key, and their values are modified using the watermark. Then, in the learning process, we iteratively update the weights and find the local minimum using a given supervised dataset. Under the assumption that the accuracy of DNN model is not sensitive to the difference in local minima, the embedding operation is performed just after each epoch so that the changed weights do not move to another point far from the initial point. Thus, our method restricts the space for finding a local minimum in order to formulate a specific formulation determined by watermark in the selected weights.

Different from the method in $[12,16]$, the loss function for watermark embedding is eliminated in the proposed method. Instead, we use the idea of the quantized index modulation (QIM) method [2] to control the amount of change due to watermark embedding to be small. After the first epoch, the changes on the watermarked weight values are corrected by performing the same embedding operation as the first epoch, which can be regarded as a restriction only on the weights selected for watermarking. Since the changes of weight values gradually converge with the progress of learning process, we can eliminate the embedding loss function.

At the learning process of DNN model, a set of supervised data is given and the loss measurements are calculated to update the weights between the nodes of neural networks. Generally, the larger the quantization step size, the larger the change in the weights due to the embedded watermark, and the more epochs are required before the training of the DNN model converges. We quantitatively evaluate the performance and training efficiency conditioned on the strength of the watermark by statistically analyzing the energy of the watermark signal. We also evaluate the robustness against pruning of DNN models with respect to the watermark strength. Under our experimental condition, it is revealed that the dominant factor is the embedding operation to the initial weight values.

\section{PRELIMINARIES}

In this section, we briefly review deep neural networks and watermarking techniques that are closely related to our research.

\subsection{Fine-Tuning}

Deep learning is a type of machine learning technique that learns high-dimensional data and extracts hidden tends from training data without handcrafting feature representations [9]. Deep neural networks (DNN) consist of basic neural network units such as linear perceptrons, convolution, and non-linear activation functions. The network units are organized as layers and are trained to recognize complex concepts directly from raw data. Although some programming frameworks are available, considerable expertise is required to create a well-designed model.

Even if a well-designed model is presented, training the model is a time-consuming task. One promising approach is to use a publicly available pre-trained models. A pre-trained model is stored network that has been trained in advance on a large dataset, typically a large image classification task. When a model is trained on a large, general dataset, this model effectively acts as a generic model. Instead of training a large model from scratch on a large dataset, it is reasonable to start training with a generic model. In case of image classification, the convolutional network layer generally contains features that are useful for the classification task. However, the fully-connected(FC) layer of a pre-trained model is specific to the original image classification task and then to the set of classes for which the model was trained. To make use of the convolutional network layer, the FC layer of the pre-trained model is replaced by a new one designed for the new classification task in the transfer learning. In addition to the replacements, some of the top layers will be frozen and the remaining layers and added FC layer will be trained in the fine-tuning.

\subsection{Conventional Works}

Watermark information is embedded inside the model (e.g., weights, activation maps) so that it can be extracted later. This technique can be categorized into two types [3]. One is a white-box watermarking method, in which the internal details are exposed to the public. The other is a black-box watermarking method where the owner of the model only has API access to the remotely deployed model. This assumes that a query is sent to verify the watermark and the corresponding output is received. It is common that the stolen model is deployed remotely without revealing its architecture or parameters. In this respect, the black-box watermarking method is suitable for realistic scenarios. However, embedding watermark is equivalent to creating a backdoor into the model, which may cause trouble for security reason.

The first white-box method was developed by Uchida et al. [12, 16]. Rouhani et al. [14] presented an improved version of it, and their research group proposed an application of fingerprinting to track illegal users. For a given DNN model, a bit string of watermark information is embedded in one or more network layer parameters. Considering the performance degradation of the model, the above conventional methods avoid directly modifying the parameters for embedding the watermark. Therefore, in order not to impair the performance of an original DNN model in its original task, a binary cross entropy is introduced in the cost function in learning process to regularize the watermark embedding task.

Watermark information is denoted by a vector $w$ of length $k$. Let $X \in \mathbb{R}^{k \times n}$ be a matrix to be kept secret, and $\boldsymbol{p}$ be vector of weights in network layers to be watermarked which length is $n$. Then, the binary cross entropy $H(\boldsymbol{p})$ is defined by

$$
H(\boldsymbol{p})=-\sum_{i=1}^{k}\left(w_{i} \log \left(y_{i}\right)+\left(1-w_{i}\right) \log \left(1-y_{i}\right)\right),
$$

where

$$
y_{i}=\operatorname{Act}\left(\sum_{j=0}^{n} X_{i j} p_{j}\right)
$$

and Act() is an activation function like a sigmoid function. Each watermark bit $w_{i}$ is embedded so that the following equation becomes true.

$$
w_{i}= \begin{cases}1 & y_{i} \geq 0.5 \\ 0 & y_{i}<0.5\end{cases}
$$

The cost function is composed of two functions as follows:

$$
E(\boldsymbol{p})=E_{0}(\boldsymbol{p})+\lambda H(\boldsymbol{p}),
$$

where $E_{0}(\boldsymbol{p})$ is the original cost function, and $H(\boldsymbol{p})$ is a regularization term that imposes a certain restriction on parameters $\boldsymbol{p}$, and $\lambda$ is an adjustable parameter. 
The embedding matrix $X$ is considered as a secret key and needs to be generated carefully so that the distribution of watermarked weight parameters becomes unnatural. Wang et al. [17] pointed out the problem of significant differences in the distribution from the watermarked parameters and presented a method to remove the watermark by overwriting attack. The main reason of this problem is that the above method changes the weights significantly in order to satisfy the condition given by Eq.(3).

\section{PROPOSED METHOD}

Conventional methods use a loss function to embed watermark in the learning process. Unlike this method, we propose a digital watermarking method that has less impact on model parameters of a DNN model by applying the QIM method [2].

\subsection{Overview}

Many DNN-based systems are created using the fine-tuning technique from a basic architecture, rather than being designed from scratch. In particular, convolutional neural networks(CNN), such as AlexNet [11], VGG [15] and ResNet [10], and Xception[5], are pre-trained with their own models. The convolutional layers can be considered as a common operation to extract features from a given multimedia content. By replacing the last few parts of the network layers, including the FC layers, a new CNN-based model architecture has been designed to accelerate the speed of system development. In addition, several common architectures have been released that follow the idea of open source software. Some of them have been released in public database such as GitHub. In such cases, the common architecture is not subject to protection.

In this study, we assume a fine-tuning model of VGG16 [15]. An overview of the proposed method is shown in Fig. 1. Based on a secret key, we sample $n$ weight parameters from $N(\gg n)$ candidates in the FC layer. The embedding operation is explained below.

(1) Select $n$ weights from the FC layer based on a secret key key, which is denoted by a vector $f$ :

$$
f=\left(f_{0}, f_{1}, \ldots, f_{n-1}\right) .
$$

(2) Perform the discrete cosine transform (DCT) on the vector $f$ to obtain the frequency components $F$.

(3) For each bit of watermark $\boldsymbol{w}$ :

$$
\boldsymbol{w}=\left(w_{0}, w_{1}, \ldots, w_{k-1}\right),
$$

modify the corresponding frequency component $F_{i+1},(0 \leq$ $i \leq k-1)$.

$$
\overline{F_{i+1}}=\operatorname{Embed}\left(F_{i+1}, w_{i}, \delta\right),
$$

Here, Embed() is an embedding function described in Sect. 3.4. Note that the DC element $F_{0}$ is avoided for embedding.

(4) Perform the inverse DCT on the vector $\overline{\boldsymbol{F}}$ and replace $f_{i}$ with the watermarked weight $\bar{f}_{i}$ of the FC layer.

If $k \ll n$, then the signal embedded as watermark is widely spread over $n$ sampled weights, which means that its effect is widely diffused. In addition, the number of candidate samples $N$ becomes much larger than $n$, which makes it difficult to analyze the existence of hidden messages from the observation of FC layer weights. Even if the $n$ samples are identified, the direct modification of the

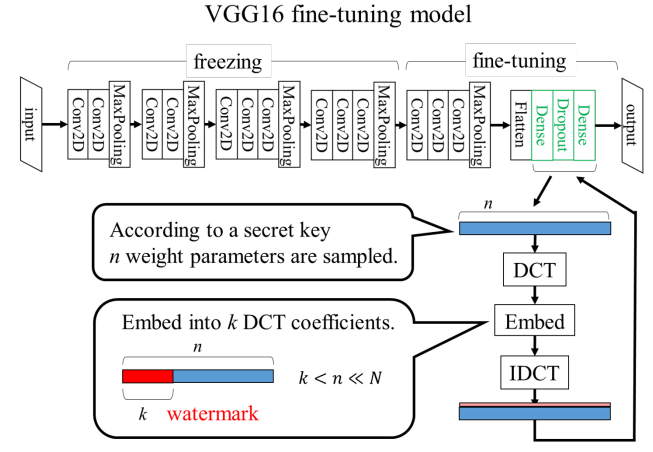

Figure 1: Embedding operation in a proposed method.

embedded watermark is difficult because of the lack of information about the frequency components $\overline{F_{i}}, 1 \leq i \leq k$. Each bit of watermark signal is embedded into some samples in a spread form in the technique of spread transform dither modulation [2], while the proposed method mixs $k$-bit watermark signal over $n$ samples. The technique has an analogue to that of orthogonal frequency division multiplexing (OFDM), and its advantage is the power efficiency and bandwidth efficiency. It is noted that we can use any orthogonal transformation instead of DCT to calculate the frequency components.

\subsection{Convergence of Embedding Operation}

In a learning process, model parameters are trained from scratch to find a local minimum. This means that the initial values of the weight parameters of the FC layer are determined randomly by default. Assuming that there is no significant difference between local minima, the selection of initial values is used for watermark embedding.

In the embedding operation described above, the initial values are changed to embed watermark. Since the values of watermarked weight parameters are slightly changed as the learning process progresses, noise is considered to be added to the watermark signal at each epoch. Hence, at each epoch, after updating the weight parameters, the embedding operation is performed to remove the noise on the watermark signal.

Remember that the number of sampled weight parameters $n$ is much smaller than the total number $N$, and a $k$-bit watermark is embedded into the $k$ DCT coefficients of the $n$ samples. Similar to the convergence of the learning process, the amount of change in the watermarked DCT coefficients also gradually decreases as the learning process progresses. Therefore, the amount of change due to the watermark embedding will converges.

At the first embedding operation to the initial weight values, the amount of change caused by the watermark embedding must be largest in the remaining processes, though it is controlled to be much smaller than the energy of sampled weight parameters. After the first embedding operation, all weights in a model are updated in the learning process to reach a local minimum. Then, after the next epoch of learning process, the same embedding operation is performed to correct the values of sampled weights because of the slight change caused by the training. Here, it is noticed that the 


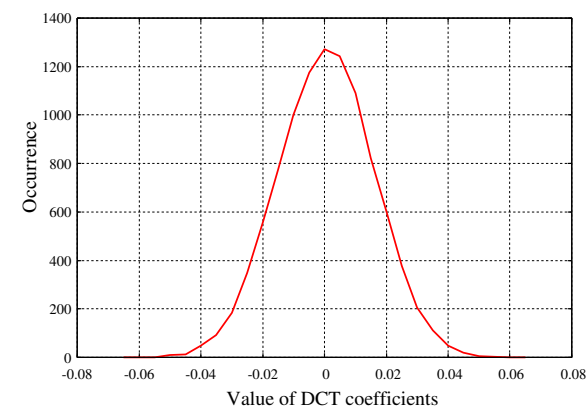

Figure 2: Histogram of frequency components of 10000 initial weights in a FC layer, where $n=2048$ and $N=8192 \times 256$.

change at the second embedding operation is much smaller than the change at the first operation. Similarly, the change in the weights becomes smaller after several epochs and is expected to converge to zero as the training progresses. In other words, the above process does not require a loss function to embed the watermark.

\subsection{Statistical Distribution of Sampled Weights}

By random sampling of weight parameters in the embedding process, the statistical distribution of their frequency components is expected to be modeled as a Gaussian distribution with zero mean because the sampled values are regarded as i.i.d random variables. In a preliminary experiment, we measured the histograms of the DCT coefficients of the weights sampled from the FC layer with randomly given initial values of the fine-tuning model. We selected 2048 weights from $8192 \times 256$ paths in the FC layer and performed the DCT in the experimental setup. By varying the initial values, a total of 10000 DCT coefficients are observed and represented in the histogram. The result is shown in Fig. 2, where the mean is 0.000485 and the variance is 0.000239 . This histogram can be regarded as a Gaussian distribution with zero mean.

Because of the zero mean of the distribution, we avoid the modification of DC component for the watermark embedding.

\subsection{Embedding Function}

In the well-known QIM method [2], the amount of change caused by the embedding process can be statistically estimated, and it has been proven to be minimum. Therefore, the impact on the accuracy of DNN models is expected to be smaller than the other watermarking methods. In order to make the existence of watermark information difficult to detect, the watermark information should not be directly embedded in the weights. According to a secret key, $n$ weights are randomly selected and their frequency components are calculated by DCT in the proposed method.

Among the $n$ frequency components, we select $k$ DCT coefficients $F_{i+1},(0 \leq i \leq k-1)$, and embed a $k$-bit watermark so that the change for the selected weights is diffused. Assuming that the value of $F_{i}$ is a random number, when it is quantized with a step size $\delta$, the amount of change varies in the range $[-\delta, \delta]$. Therefore, the expected energy of change $\epsilon$ can be calculated by the following equation.

$$
\epsilon=\frac{1}{2 \delta} \int_{-\delta}^{\delta} t^{2} d t=\frac{\delta^{2}}{3}
$$

Hence, the total energy $E_{w}$ of $k$-bit watermark can be given as follows:

$$
E_{w}=\frac{k \delta^{2}}{3} .
$$

After the inverse DCT, the energy is spread over $n$ samples. Thus, the expected watermark energy at each sample is $E_{w} / n$.

As analyzed in Sect. 3.3, the statistical distribution of the DCT coefficients $F_{i+1}$ should be considered so that the watermarked coefficients $\bar{F}_{i+1}$ are not biased. Since the histograms of DCT coefficients is symmetrically distributed with respect to zero point, the region for quantization needs to be adjusted to take into account this statistical property. Based on these considerations, the embedding function is defined as follows.

$\operatorname{Embed}(F, w, \delta)= \begin{cases}\delta \cdot\left\lfloor\frac{x-\frac{\delta}{2}}{\delta}\right\rfloor+\frac{\delta}{2} & \text { if }\left\lfloor\frac{x-\frac{\delta}{2}}{\delta}\right\rfloor \bmod 2=w, \\ \delta \cdot\left(\left\lfloor\frac{x-\frac{\delta}{2}}{\delta}\right\rfloor+1\right)+\frac{\delta}{2} & \text { otherwise. }\end{cases}$

where $\lfloor\cdot\rfloor$ stands for the floor function.

The energy of the watermark can be controlled by setting the bit length $k$ and the quantization step size $\delta$ of the watermark. The larger the watermark energy, the longer it will take to converge after training the model. The embedding of the watermark is considered to be shifted from the initial point to one of the nearest local minima, which makes the analysis of the watermark more difficult.

\subsection{Extraction}

To extract the watermark, the same $n$ weight parameters are selected from the FC layer based on the secret key. Then, the $k$ DCT coefficients $F_{i}^{\prime},(1 \leq i \leq k)$ are checked by the following equation.

$$
\begin{aligned}
w_{i}^{\prime} & =\operatorname{Extract}\left(F_{i+1}^{\prime}, \delta\right) \\
& =\left\lfloor\frac{F_{i+1}^{\prime}}{\delta}\right\rfloor \bmod 2
\end{aligned}
$$

From an attacker's point of view, it is difficult to add noise to only $k$ DCT coefficients without a secret key, because there is no information about the selection of $n$ weights from the huge number of $N$ candidates in the FC layer as well as their order among the selected weights. Even if some of the sampled weights happen to be modified by adding noise, the noise energy will be spread over all DCT coefficients $\overline{\boldsymbol{F}}$. It is difficult for an attacker to remove/modify the watermark by adding noise without seriously degrading the performance of model.

\section{EXPERIMENTS}

We implemented the proposed method and conducted experiments to evaluate the performance in terms of test accuracy, accuracy and loss value during training, and robustness against pruning attacks. Since the learning process starts with scratch, the results may vary depending on the initial settings. Therefore, in this experiment, 10 trials were conducted and the average values are calculated. 
Table 1: SNR of watermarked weights at the 1st epoch.

\begin{tabular}{|c|c|c|c|c|}
\hline$\delta$ & 0.005 & 0.01 & 0.02 & 0.04 \\
\hline SNR $[\mathrm{dB}]$ & 26.626 & 20.605 & 14.585 & 8.564 \\
\hline
\end{tabular}

\subsection{Experimental Conditions}

In this study, we used the VGG16 [15] as a pre-trained model, which was developed by Visual Geometry Group of Oxford University. This model was trained using over 1 million images from the ImageNet [8] database. Based on this pre-trained model, we fine-tuned the VGG16 model by implanting new FC layers after the final convolutional layer. The number of nodes at the final convolutional layer is $8192(=4 \times 4 \times 512)$ in VGG16, and these nodes are connected to the new FC layers with 256 nodes. The number of candidates for selecting weights from the first FC layer is more than 2 millions, $N=8192 \times 256$. From such a huge number of candidates, we select only $n=2048$ weights according to a secret key and embed watermark of $k=128$ bits, which is a random binary sequence in this experiment.

The 17 Category Flower Dataset [13] provided by Visual Geometry Group of Oxford University was used as the dataset for training the fine-tuning model. Training is performed by dividing the 17 classes into $70 \%$ training data, $10 \%$ validation data, and $20 \%$ test data.

\subsection{Strength of Watermark Signal}

If the variance of weights sampled from a FC layer with the above setting is $\sigma^{2}$, then the energy of the weights is $n \sigma^{2}$. According to the statistical analysis in Sect. 3.3, the variance of the DCT coefficients of the weights is 0.000485 , so the total energy of the sampled weights (host signal) is $E_{f}=0.000239 \times 2048 \approx 0.49$. From Eq.(9), the total energy of the 128 -bit watermark is $E_{w}=128 \delta^{2} / 3$. The SNR, which is considered to be the ratio of the host signal to watermark, can be estimated for a given quantization step $\delta$.

$$
S N R=10 \log _{10}\left(\frac{E_{f}}{E_{w}}\right)
$$

Table 1 enumerates some examples of $S N R$ for given $\delta$.

The SNR is the ratio between the initial values of the weights given in the programming code and the watermarked ones. Since the initialization process in the proposed method can be interpreted as the generation of watermarked initial values of the weights, which are specified by watermark, the impact on the performance of the model should be small. As the learning process progresses, the model converges to a local minimum close to the watermarked initial point. In the embedding operation every after each epoch, the change in the watermarked weights is corrected so that the watermark can be extracted.

Figure 3 shows the change in the weight values due to the embedding operation in terms of mean squared error (MSE). Due to the initialization process described above, we can see a strong peak at the first epoch. The figure also indicates that the MSE gradually converges as the training progresses, confirming the irrelevance of the embedding loss function. As 128-bit watermark signal is spread over $n=2048$ weights, the total energy is $E_{w}=2048 \times$ MSE. After the second epoch, the MSE gradually decreases from the range

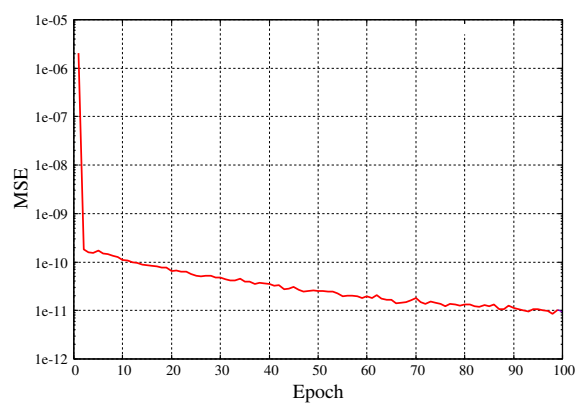

Figure 3: Amount of change in weights caused by the embedding operation at each epoch when $\delta=0.01$.

Table 2: Comparison of test accuracy and loss.

\begin{tabular}{|c|c|c|c|c|c|}
\hline & & \multicolumn{4}{|c|}{ step size $\delta$} \\
\cline { 3 - 6 } & original & 0.005 & 0.01 & 0.02 & 0.04 \\
\hline accuracy & 0.897 & 0.895 & 0.896 & 0.895 & 0.901 \\
\hline loss & 0.358 & 0.345 & 0.377 & 0.381 & 0.359 \\
\hline
\end{tabular}

$2 \times 10^{-10}$ to $1 \times 10^{-11}$ which SNR varies in the range from 60.8 to $78.8[\mathrm{~dB}]$. As the similar tendency is observed when the step size $\delta$ is varied, the details of the data is omitted.

In the proposed method, the selection of $n$ weights among $N$ candidates is kept secret. Without the secret key, an attacker has to observe the values of $N$ weight and guess the watermarked weights. Since the values of the weights fluctuate, it is difficult for the attacker to analyze the tiny change caused by the watermark embedding.

\subsection{Performance of DNN Model}

We evaluate the performance of watermarked model in terms of accuracy and loss measurements. Table 2 enumerates the test accuracy and loss after 100 epochs with varying step size $\delta$. Figure 4 and Figure 5 show the training progress. The results show that there is no significant difference in the performance of the watermarked model.

In the proposed method, watermark is inserted at the initialization of weight values of a DNN model, and the embedding function is used to compensate for the change at each epoch of the training. The learning process can be interpreted as a training with restrictions on some weights in the model. Since a local minimum is searched from the initial values in the learning process, the change at each epoch is significantly smaller than the change at the initial setting. Figure 4 and Figure 5 confirms these features of the proposed embedding operation.

\subsection{Robustness}

We evaluate the robustness against the pruning attack that removes paths with small weight values. The higher the pruning rate, the more difficult it is to extract the watermark because the randomly selected weight values will contain small weight values.

At each epoch in the learning process, the watermarked initial weight values are slightly changed and are corrected by using the 


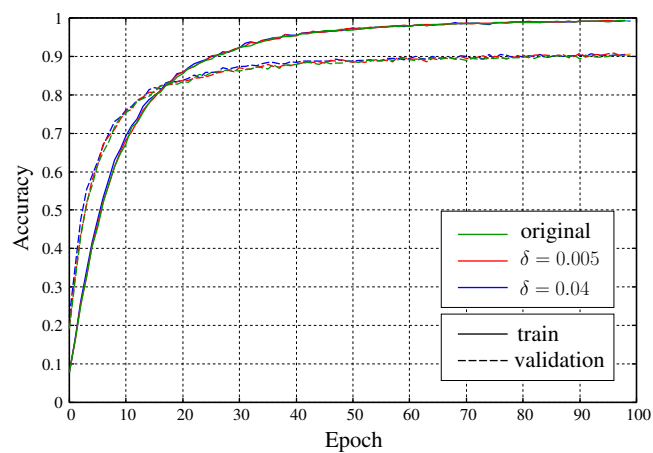

Figure 4: Accuracy at each epoch.

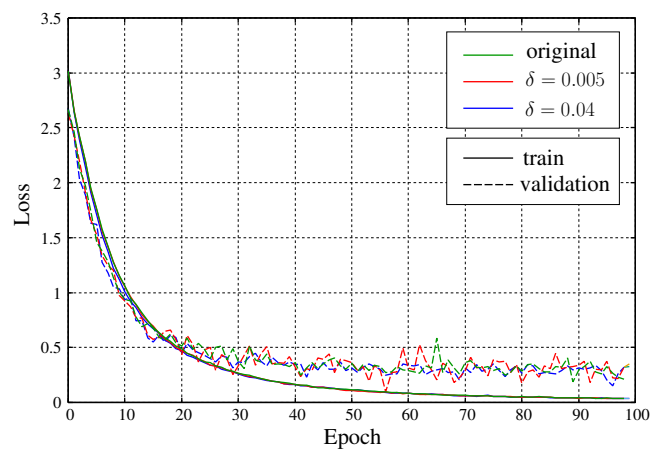

Figure 5: Loss at each epoch.

embedding operation. Assuming that the amount of change at each epoch is small, we omit the embedding operation at each epoch. The change at each epoch of learning process can be regarded as noise added to the watermarked initial weight values, which reduces the robustness against attacks. Figure 6 compares the robustness of the "all" method for performing the embedding operation at each epoch and the "first" method which performs the embedding operation only at the initial setting. It can be seen that there is no significant difference in the error rate for "all" and "first". It means that the embedding operation to the initial weight values is the dominant factor in the proposed method in this experimental condition. However, it does not confirm the irrelevance of the embedding operation at each epoch. Thus, the proposed method needs to be evaluated using several fine-tuning models whether the modification of initial weight parameters is sufficient for the watermark embedding. It is also noted that the robustness against the pruning attack can be improved by increasing the step size $\delta$ because the strength of the watermark signal relatively small in our experiments.

\section{CONCLUSIONS}

In this paper, we proposed a white-box watermarking method for the FC layer of fine-tuning model that has little effect on the performance of model. The embedding operation is performed on the frequency components of the sampled weights so that the signal embedded as watermark can be diffused over the samples. One of the advantages of this method is that the distortion level is small

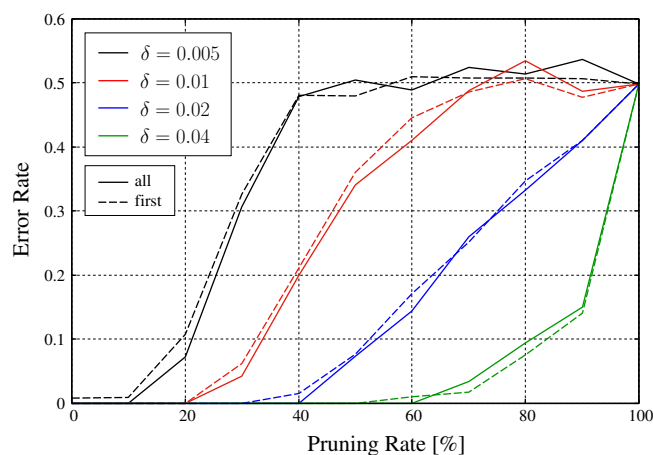

Figure 6: Robustness against the pruning attack.

and can be controlled by setting a quantization step size. Without the use of the embedding loss function, the change caused by the embedding operation converge as the learning process progresses.

\section{ACKNOWLEDGMENTS}

This research was supported by the JSPS KAKENHI Grant Number 19K22846, JST SICORP Grant Number JPMJSC20C3, and JST CREST Grant Number JPMJCR20D3, Japan.

\section{REFERENCES}

[1] Y. Adi, C. Baum, M. Cisse, B. Pinkas, and J. Keshet. 2018. Turning Your Weakness Into a Strength: Watermarking Deep Neural Networks by Backdooring. In 27th USENIX Security Symposium (USENIX Security 18). 1615-1631.

[2] B. Chen and G. Q. Wornell. 2001. Quantization index modulation: a class of provably good methods for digital watermarking and information embedding. IEEE Trans. Inform. Theory 47, 4 (2001), 1423-1443.

[3] H. Chen, B. D. Rouhani, X. Fan, O. C. Kilinc, and F. Koushanfar. 2018. Performance Comparison of Contemporary DNN Watermarking Techniques. CoRR abs/1811.03713 (2018). arXiv:1811.03713 http://arxiv.org/abs/1811.03713

[4] H. Chen, B. D. Rouhani, C. Fu, J. Zhao, and F. Koushanfar. 2019. DeepMarks: A Secure Fingerprinting Framework for Digital Rights Management of Deep Learning Models. In Proc. ICMR'19. 105-113.

[5] F. Chollet. 2017. Xception: Deep learning with depthwise separable convolutions. In Proc. of CVPR'17.

[6] A. Choromanska, M. Henaff, M. Mathieu, G. B. Arous, and Y. LeCun. 2015. The loss surfaces of multilayer networks. Artificial Intelligence and Statistics.

[7] Y. N. Dauphin, R. Pascanu, Ç. Gülçehre, K. Cho, S. Ganguli, and Y. Bengio. 2014. Identifying and attacking the saddle point problem in high-dimensional nonconvex optimization. In Proc. NIPS'14. 2933-2941.

[8] J. Deng, W. Dong, R. Socher, L. Li, Kai Li, and Li Fei-Fei. 2009. ImageNet: A large-scale hierarchical image database. In Proc. CVPR'09. 248-255.

[9] I. Goodfellow, Y. Bengio, and A. Courville. 2016. Deep Learning. MIT Press.

[10] K. He, X. Zhang, S. Ren, and J. Sun. 2016. Deep residual learning for image recognition. In Proc. of CVPR'16.

[11] A. Krizhevsky, I. Sutskever, and G. E. Hinton. 2012. ImageNet classification with deep convolutional neural networks. In Proc. of NIPS'12.

[12] Y. Nagai, Y. Uchida, S. Sakazawa, and S. Satoh. 2018. Digital watermarking for deep neural networks. International fournal of Multimedia Information Retrieval 7 (2018), 3-16.

[13] M.-E. Nilsback and A. Zisserman. 2006. A Visual Vocabulary for Flower Classification. In Proc. CVPR'06, Vol. 2. 1447-1454.

[14] B. D. Rouhani, H. Chen, and F. Koushanfar. 2019. DeepSigns: An End-to-End Watermarking Framework for Ownership Protection of Deep Neural Networks. In Proc. ASPLOS'19. 485-497.

[15] K. Simonyan and A. Zisserman. 2015. Very deep convolutional networks for large-scale image recognition. In Proc. of ICLR'15.

[16] Y. Uchida, Y. Nagai, S. Sakazawa, and S. Satoh. 2017. Embedding watermarks into deep neural networks. In Proc. ICMR'17. 269-277.

[17] T. Wang and F. Kerschbaum. 2019. Attacks on digital watermarks for deep neural networks. In Proc. ICASSP'19. 2622-2626.

[18] J. Zhang, Z. Gu, J. Jang, H. Wu, M. Ph. Stoecklin, H. Huang, and I. Molloy. 2018. Protecting intellectual property of deep neural networks with watermarking. In Proc. ASIACCS'18. 159-172. 